\title{
Commission 26: Double and Multiple Stars
}

\author{
PRESIDENT: William I. Hartkopf \\ VICE-PRESIDENT: Christine Allen \\ ORGANIZING COMMITTEE: J.A. Davis, F.C. Fekel, P. Lampens, \\ J.F. Ling, E. Oblak, T.D. Oswalt, \& C. Scarfe (past president)
}

\section{Introduction}

While Commission 26 remains one of the smallest in the IAU, it maintains an active program which belies its size, as highlighted below. Further information on the commission may be found at our website: http://ad.usno.navy.mil/wds/dsl.html\#iau. This site includes links to other sites and to major databases and catalogues, as well as bibliographies of recent double star papers, an archive of the Commission's Information Circulars, and a list of upcoming meetings.

\section{Meetings}

\subsection{IAU Colloquium 191}

The most notable achievement of the past triennium was IAU Colloquium 191, The Environment and Evolution of Binary and Multiple Stars. This meeting was sponsored by Commission 26, with the support of Commissions 30, 34, 37 and 42; Division IV was the coordinating division. The meeting was held in honor of one of the pioneers of Mexican astronomy, Arcadio Poveda, and took place in the city of his birth, Mérida, Yucatán, in February, 2003. The principal sponsor within Mexico was the Universidad Nacional Autónoma de México, Instituto de Astronomía. The scientific organizing committee was chaired jointly by Christine Allen and Colin Scarfe, the local organizing committee by Christine Allen.

The meeting was attended by about eighty people from twenty-four countries, and continued a long tradition of successful topical meetings organized by Commission 26 . Its topic was in part a sequel to IAU Symposium 200, which concentrated on binary star formation, extending to the role binaries' environment plays in their subsequent evolution, and how they in turn affect that environment. A significant type of environment is that of a star cluster, and for clusters the meeting was a sequel to one that took place in Calgary, Canada in 1995. Now, of course, that subject has been extended with the detection of numerous binaries in nearby galaxies by means of major surveys or as byproducts of gravitational micro-lensing studies. Moreover, we are now discovering low-mass objects such as planets within binary systems; part of the meeting was concerned with their role.

The meeting was organized around eighteen invited talks, with contributed papers related to them presented either orally or as posters. Ample time was provided for poster viewing, and for discussion following each oral presentation. The difficult task of summing up was ably handled, with wit and humor, by Edward Guinan.

Proceedings of the meeting were edited by Christine Allen and Colin Scarfe, and published as Volume 21 of Revista Mexicana de Astronomía y Astrofísica, Serie de Conferencias. 


\subsection{Multiple Stars Workshop}

A workshop Multiple stars across the H-R diagram was organized by ESO and held in the summer of 2005 in Garching, Germany. It attracted 41 participants and concentrated on stellar systems with 3 and more components. Studies of young multiples and their formation mechanisms were at the focus of the Workshop, but other subjects included evolution of multiple systems and their components, special objects, statistics, observing techniques, and future programs. The meeting showed the relation of multiple-star studies to other areas of astrophysics such as stellar formation and evolution, planets, X-ray astronomy, etc., and the general importance of continuing research on multiple stars.

\subsection{Dubrovnik Workshop}

Significant developments on observational techniques were presented during the workshop Spectroscopically and spatially resolving the components of close binary stars, held in October 2003 in Dubrovnik. These developments concern, among other things, the very high angular resolution work on the orbits of binaries which gives a bridge between the traditional division of the closer spectroscopic and eclipsing binaries and the wider visual binaries. Other interesting techniques are adaptive optics imaging to complete orbits and speckle interferometry for angular separation to $0^{\prime \prime}$. 1 . We can also mention the (very) longbaseline interferometer to be able to separate components close to a few mas (Hummel et al. 2004) and combinations of astrometry with spectroscopy (Pourbaix et al. 2004) or optical interferometry with lunar occultation (Richichi 2004). These new observational developments at the boundaries of the visual binary field, permit a better knowledge of the mass-luminosity relation based on visual binaries at the end of the main sequence (Henry 2004) and an advance on the formation model of the double and multiple systems.

The cross-correlation techniques applied to the determination of radial velocities of close double stars permits also the discovery of new triple systems, in the frontier of the visual multiple stars field. This technique, used in the frame of an international collaboration, is pertinent to obtain high quality masses of all components of the systems and permits the study of some aspects in the stellar evolution (Oblak et al. 2004).

\subsection{Michelson Interferometry Summer School/Workshop}

Workshops are organized each summer by the Michelson Science Center and usually held on the Caltech campus in Pasadena, CA. The 2002 and 2003 meetings concentrated on interferometry, with the 2004 meeting highlighting coronography and the 2005 meeting astrometry (with an "astrometry basics" seminar held at Yale University the previous week). These workshops are intended mainly for graduate students and postdoctoral researchers, and include tutorial lectures, informal conversation, and site visits to nearby interferometer facilities. Although these week-long events concentrate on equipment and techniques as they can be specifically applied to the challenge of extra-solar planet detection, ancillary astrophysical topics, such as the high-precision characterization of binary stars, figure prominently in the curricula associated with each school and workshop.

\subsection{Symposium 240}

During this triennium we successfully proposed a symposium entitled Binary Stars as Critical Tools and Tests in Modern Astrophysics, sponsored by Commissions 26 and 42 with support from 11 other commissions and working groups. This 3.5-day meeting will be held in conjunction with the $26^{t h}$ General Assembly in Prague, in August 2006. 


\section{Information Circulars}

For many years the commission has published information circulars three times annually. Begun by Paul Muller and continued by Paul Couteau, the circulars are currently edited by J.A. Docobo and J.F. Ling of the Observatorio Astronómico "R.M. Aller" at the Universidad de Santiago de Compostela, Spain. Circulars are distributed to all commission members electronically, and also archived on the commission website. Ten circulars, numbered 147 to 156 inclusive, were published during the past triennium. Their contents are summarized below; see the website for a more thorough report.

New orbits and newly discovered double stars: A total of 185 new orbits were announced, 119 of them for objects north of the celestial equator, and 66 for southern systems. Numerous authors contributed orbits, with some appearing in every circular. Circular 156 listed discovery of a new system and \#149 the rectification of another.

Publication lists: Circulars 149, 152 and 155 included lists of papers on double stars published in 2002, 2003 and 2004 respectively.

Obituaries: Circulars 155 and 156 carried obituaries of Geoffery G. Douglass and Richard L. Walker, respectively, two distinguished former members of the commission.

Miscellaneous announcements and reports: Several circulars contained announcements, whose subject matter ranged from the publication of new catalogues, and revisions to older ones, to information on new telescopes and instruments. New amateur observing efforts were noted in Circulars 148 and 155, and a report of the Commission's business meeting at the Sydney GA was published in Circular 151.

\section{Catalogues and Journals}

The U.S. Naval Observatory double star program maintains four astrometric and photometric catalogs, each of which has seen considerable growth during the past three years. These four catalogs are updated nightly on the USNO website.

The Washington Double Star Catalog is the principal repository for all published astrometry of visual binary and multiple stars. As of July 2005 the WDS contained about 710,000 mean measures of over 100,000 systems, a $20 \%$ increase in number of means in the past three years. The database also continues to be improved through correction of errors, removal of duplicate discovery designations and/or measures, determination of precise coordinates (completed at present for $>96 \%$ of the systems), and determination of secondary proper motions. Observing lists are provided on demand for other observers.

As of July 2005, the Sixth Catalog of Orbits of Visual Binary Stars, included 1,850 orbits of 1,750 systems. The catalog was recently modified to include published formal errors, and reformatted to accommodate short-period orbits of close interferometric pairs.

The Fourth Catalog of Interferometric Measurements of Binary Stars currently includes 131,101 observations of 67,943 systems, increases of roughly 30\% since July 2002 .

The Second Photometric Magnitude Difference Catalog has grown by roughly a factor of three during the past triennium, and now includes 202,276 measures of 62,620 systems. Much of this increase has come though matches against the WDS and 2MASS databases.

An internal catalog of rectilinear elements has been created, with the aim of eventually posting it to the web as well. Recent studies have shown that these linear fits often yield more precise differential proper motions than are available elsewhere, due to the long time base of differential astrometry measures available in the WDS database. 
The Catalogue of Orbits of Visual Double Stars and their Ephemerides, complementary to the USNO catalogues because of its somewhat different selection criteria, is maintained at the Universidad de Santiago de Compostela. Updates are posted regularly on their website and linked to the Commission's webpage.

The Journal of Double Star Observations is a new web-based quarterly journal, begun in early 2005 by astronomers at the University of South Alabama. This page-charge free electronic-only publication is intended as a venue for both professionals and amateurs.

\section{Speckle interferometry and other single-aperture high-resolution astrometry techniques}

Several astrometry projects using speckle interferometry or adaptive optics were initiated or continued during the past triennium, as these techniques largely supplanted micrometry for obtaining differential astrometry of visual binaries.

E. Horch, W. van Altena, and collaborators have maintained an active speckle program using primarily the Wisconsin-Indiana-Yale-NOAO (WIYN) 3.5-m at Kitt Peak, Arizona. Their work in the last three years has focused primarily on characterization and use of CCD-based speckle imaging with the RIT-Yale Tip-tilt Speckle Imager (RYTSI), as well as follow-up observations of double stars discovered by Hipparcos.

RYTSI is a unique speckle imager that uses a large format CCD as a memory cache of many speckle images prior to full-frame readout. Through the use of two galvanometric scanning mirrors, the star image is moved in a serpentine step-and-expose pattern, with each exposure representing one speckle pattern. The RYTSI instrument was completed in June 2001, and has been successfully used at the WIYN Telescope on ten observing runs. The instrument was recently coupled with the Kitt Peak Mini-Mosaic CCD imager at WIYN, where as many as 900 speckle patterns were recorded before full frame readout of the chip. The method is reasonably efficient at 8-m and smaller class telescopes given current CCDs, and is compatible with improvements in CCD readout noise and speed.

CCD-based speckle has also been shown to retain magnitude difference information about close binaries, something that has proven elusive with intensified-CCDs. The UMass-Yale group has used the CCD method to measure $\Delta \mathrm{m}$ 's of a number of subarcsecond binary stars, many of which were discovered by Hipparcos. The results so far are in reasonably good agreement with Adaptive Optics results of ten Brummelaar and collaborators. This gives some real hope that an increasing number of "speckle" binaries will soon have well-determined magnitude differences in multiple bandpasses in addition to the precise relative astrometry for which speckle is famous. This would be an important step forward in using these stars as tests of stellar structure and evolution.

PISCO, another speckle camera dedicated to the study of binary stars, has been operational since January 2004 on the 1-meter Zeiss telescope of I.N.A.F.- Osservatorio Astronomico di Brera at Merate, Italy. PISCO was designed and built at Midi-Pyrénées Observatory (Toulouse) in the early 1990's and used from 1993 to 1998 at the 2m Bernard Lyot telescope of the Pic du Midi Observatory. Due to a change of policy by the timeallocation committee, it was no longer used after 1998 for regular observations of visual binaries. A group of European astronomers involved in the study of binary stars with PISCO decided to look for a new host telescope on which PISCO could be mounted and operated on a regular basis. In November 2003, PISCO and the intensified CCD camera of Nice University were successfully installed at the Cassegrain focus of the Zeiss telescope in Merate and after a few weeks of tests it became fully operational in 
January 2004. Subsequent observations confirmed the possibility of observing visual binaries with separations down to $0^{\prime \prime} .14$ and with luminosity differences up to 4 magnitudes. Two papers (Scardia et al. 2005a, 2005b) have already resulted from the observations of 2004.

The speckle interferometry program also continues at the USNO. Most observations have been carried out on the USNO 26-inch refractor, with nearly 4,200 measures published or in press since mid-2002 (c.f., Mason et al. 2004a, 2004b), despite suspension of observing during much of 2004 as the historic Clark refractor underwent extensive electrical and mechanical upgrades. Much of this observing effort has been directed toward duplicity confirmations and observations of systems not measured for many years, as well as regular monitoring of potential orbit systems. The speckle camera continues to be used at other telescopes as well, including the KPNO and CTIO 4-meters and the $1.5 \mathrm{~m}$ at the Naval Observatory's Flagstaff Station.

In the past triennium, adaptive optics (AO) has produced impressive new results in the field of binary stars. For the first time, complete statistics of faint companions to massive (B,A-type) primaries has been pinpointed (Shatsky \& Tokovinin 2002, Kouwenhoven et al. 2005). Binarity of M-dwarfs and brown dwarfs has been probed (e.g., Siegler et al. 2005) and revealed a telltale story of their formation. Bouy et al. (2004) determined a first dynamical mass of an L-dwarf. Large surveys of pre-main-sequence and even protostellar (Patience et al. 2003, Duchene et al. 2004) binaries are being conducted with AO. Patience et al. (2002) combined wide-field imaging, speckle, and AO to reveal two binaries and a triple among the first sample of exoplanet systems.

A search for faint companions to $\mathrm{O}$ stars was done using the AEOS 3.6m and AO system at I band. The search included all 143 stars within known $\mathrm{O}$ star systems that were accessible to AEOS. Of the 84 stars observed thus far, 31 new and 24 previously known companions were measured, with companions found as close as $0^{\prime \prime} .34(\Delta \mathrm{m}=$ 4) and a dynamic range of 10 magnitudes (as close as $2^{\prime \prime}$; see Turner et al. 2003 and in preparation). While investigating atmospheric turbulence using $\mathrm{AO}$ and the AEOS telescope, several dozen binaries were observed at I, J, H, and K bands, reaching I-band $\Delta \mathrm{m}$ 's of $8 \mathrm{mag}$ and discovering several faint new companions to known systems (Roberts et al. 2005).

Peter Tuthill and Jamie Lloyd have begun experiments combining an aperture mask with the Palomar AO system; this technique has shown great promise in improving both astrometric and especially photometric accuracy, with order of magnitude improvements in photometric precision reported.

\section{Long-baseline interferometry}

Long-baseline optical/infrared interferometry is developing into an important tool in binary and multiple star astrophysical research and the past three years has seen major progress. Particularly notable are the increasing application of combined interferometric and spectroscopic observations to determine individual masses of component stars and accurate distances to systems, thereby enabling critical tests of evolutionary stellar models, and the development of multi-aperture imaging of multiple systems. Highlights of the application of long-baseline interferometry to binary and multiple star research include:

A program to measure PMS binary systems is being carried out with the Keck Interferometer (Boden et al. 2005) with preliminary results for the B binary system of the quadruple system HD 98800 revealing significant differences from model predictions. 
Accurate mass-luminosity and mass-radius relations for $\mathrm{M}$ dwarfs in the solar neighborhood have been determined by combining adaptive optics image, radial velocity, and ESO Very Large Telescope Interferometer (VLTI) data (Delfosse et al. 2004).

Models of the evolution of metal poor stars have been tested by the combination of interferometric and spectroscopic data for the binary HD 185987 (Torres et al. 2002).

The Sydney University Stellar Interferometer (SUSI) has been used to determine the orbit of $\beta$ Cen and, in combination with spectroscopy, the masses of the two $\beta$ Cephei components have been shown to be less than those generally accepted for early $\mathrm{B}$ giant stars. An accurate distance to the system has been determined that differs significantly from the Hipparcos value (Davis et al. 2005).

In addition to a continuing programme of interferometric orbital determinations the Palomar Testbed Interferometer (PTI) now has a differential astrometric capability and this has been used to determine the mutual inclination of V819 Herculis triple system.

The three aperture Infrared/Optical Telescope Array (IOTA) has been used to image the binary star Capella, the underlying binary of the prototypical colliding-wind source WR 140, and the Am components of $\lambda$ Vir.

The Navy Prototype Optical Interferometer (NPOI) has achieved imaging with more than three separate telescopes for the first time with six-aperture imaging of the hierarchical triple $\eta$ Vir, enabling the orbit of the close pair to be determined leading to a value for the mass ratio and the relative orbit inclination. NPOI and Mark III interferometric data have been combined with spectroscopic data to determine the distance to the binary star Atlas in the Pleiades. A survey for multiplicity amongst bright stars is being conducted with NPOI.

The CHARA Array was transformed from developmental effort to routinely operating facility during 2003-2004. A variety of observing programs have been undertaken, including oblateness and gravity darkening exhibited by rapidly rotating stars, diameters of M dwarfs, calibration of the Cepheid P-L relationship, survey of exoplanet systems for contamination by face-on binaries, stellar limb darkening, and a survey for companions to $\mathrm{O}$ stars. CHARA plans to undertake an extensive program of resolving double-lined spectroscopic binaries during 2006. In the interim, binary star studies have been limited to astrometry of "wide" binaries (separations 20-70 mas) that display separated fringe packets within a given fringe scan. Accuracies obtained thus far are approaching $25 \mu$ as. An additional application of separated fringe packets is the use of one packet to calibrate the interferometric visibility of the companion star if it is itself a spectroscopic binary; experiments are underway with the inner two subsystems of the hierarchical $\eta$ Orionis quintuple system. In 2005 a prototype multi-beam combiner developed for the Array successfully combined four beams simultaneously measuring closure phases and, for the first time by an optical interferometer, closure amplitudes. This instrument will be expanded to six-way beam combination during 2006, permitting imaging of spectroscopic binaries.

\section{Other projects}

\subsection{Orbital systems}

A unique multiple system $41+40$ Dra has been studied by a combination of spectroscopy and interferometry (Tokovinin et al. 2003). Its extremely high eccentricity $e=0.9754$ permits precise timings of periastron passages, giving hope to measure the rate of tidal circularization in the near future.

Poveda \& Allen (2004) studied the distribution of major semiaxes of visual binaries of different ages, and found that for all groups studied it is $f(a) d a \sim d a / a$, up to a 
maximum value of $a$ that depends on the age of the binary and on the conditions of the environment it has traversed.

\subsection{Trapezium systems}

Allen et al. (2004) examined the internal motions of trapezium systems. Combining observations dating back to 1830 with modern ones they found that most systems do not show significant motions. However, a few stars were found escaping from their trapezia with large velocities. These stars were identified as runaway stars recently formed by dynamical interactions.

\subsection{Young binaries}

On the basis of a broad range of high precision astrometric data (HST, speckle, lunar occultation) Tamazian et al. (2002) have for the first time calculated preliminary orbits and dynamical masses for four binary T Tau stars (V773 Tau, FO Tau, FS Tau and GG Tau Aa) as well as significantly improved that of DF Tau - the only T Tau type star with the known visual orbit prior to this determination. The obtained results allowed to compare empirically determined physical properties of $\mathrm{T}$ Tau stars with pre-main sequence stellar evolutionary models. The conclusion was made that the models of Palla \& Stahler and Siess, Dufour \& Forestini describe somewhat better the observational data.

An improved orbit for T Tau Ba,Bb system allowed Tamazian (2004) to make a first ever direct dynamical estimate for the mass of $\mathrm{T}$ Tau Ba which belongs to a rare class of known "infrared companions" (IRC) to T Tau stars. The mass of IRC was found to be $2.3 \mathrm{M}_{\odot}$, suggesting that it may be a young, heavily reddened F-K star experiencing an episode of enhanced accretion, possibly tied to its orbital phase.

\subsection{Binaries with massive components}

Discoveries of Wolf-Rayet binary and triple systems have increased markedly in the last few years. With these discoveries has arisen a broader understanding of the properties of the WR stars themselves as well as the interactions of massive stars in binary systems. Two especially noteworthy examples are the discovery of both periodic dust-emitting (Williams et al. 1987) and continuous dust-emitting (Tuthill et al. 1999) WR stars as binary systems with different orbital geometries. The dust arises in the wind-collision zone as a consequence of the high densities and low temperatures in the wind interaction zone at small ( few AU) binary separations. Many of these are not just binary, however, but multiple systems. HST observations have found extra components for many of these systems (Wallace 2003, Wallace et al. 2001). WR 48, for example, has a 19.1 day orbital period (Hill et al. 2002) with a close companion, as well as a wider companion at a 46 mas separation (Hartkopf et al. 1999, Wallace et al. 2001). While several years ago this would be thought to be an unusual system, we now know of at least 5 such triple systems (WR 48, 98a, 104, 112, 153) where all of the components can be confirmed to interact. At wider separations, HST WFPC2 observations (Wallace 2003, Wallace et al. 2005) suggest that the numbers of visual companions to WR stars are underestimated by a factor of 4-5. Undoubtedly, with the advent of higher angular resolution observations, the number of known close multiple systems will continue to grow. More of these systems are being sought, using KI, IOTA, VLTI, and HST FGS1R interferometric observations to explore binary parameter space inaccessible via either photometry or spectroscopy.

\subsection{Disentangling composite spectra}

P. Hadrava (Astronomical Institute, Czech Academy of Sciences) has developed new Fourier-based software (KOREL) to disentangle composite spectra. By comparing all 
available spectra of a system at different orbital phases, the method fits them as a superposition of some a priori unknown spectra attributed to individual components, each one Doppler shifted. The routine can handle differing line strength, and is also able to decompose telluric lines. Output may include individual radial velocities at each observed epoch or a direct solution of orbital parameters. Moreover, the component spectra, which are obtained simultaneously with the orbital parameters, can be used for subsequent interpretation of the results, e.g., for determination of spectral types.

\subsection{Stellar pulsation}

Binary and multiple stars with well-characterized components are attractive targets to study a number of different phenomena of high astrophysical interest apart from their own formation and history. This also includes stellar pulsation. Recently, an in-depth study of the triple system DG Leo AB was performed, including photometry, high-resolution spectroscopy and astrometry. The photometric study allowed the detection and precise determination of at least four pulsation frequencies of type Delta Scuti. An additional slow variation due to ellipsoidal variability of the inner binary system Aa, Ab was also reported (Lampens et al. 2005a). Application of Hadrava's spectral disentangling technique allowed to successfully disentangle the component spectra, showing that all three components have similar effective temperatures, surface gravities, ages and masses but different atmospheric chemical compositions as mild metallicity was detected in two of the three A-type stars. However, the Delta Scuti pulsating component has a solar chemical composition (Frémat et al. 2005). Using a newly obtained speckle position, a combined astrometric-spectroscopic orbit based on all astrometric and spectroscopic data known to-date has been derived (Lampens et al. 2005b). Application of the spectral disentangling technique has lately been performed with good result in a number of cases, including also several multiple systems (e.g. Hensberge et al. 2005, Koubsky et al. 2005).

\subsection{Eclipsing binaries}

Malkov (2003) and colleagues have compared radii of eclipsing binary components and single stars and found a noticeable difference for B0V-G0V components of eclipsing binaries and single stars of the corresponding spectral type. This difference can be confirmed by a re-analysis of results of other published investigations and, in particular, it can explain the disagreement between published scales of bolometric corrections.

According to their results, A- and F-type main sequence eclipsing binaries have larger radii and/or higher temperatures than single stars while B-type eclipsing binaries have smaller radii. In proposing explanations for these features they have concluded that the mass-luminosity relation based on empirical data of eclipsing binary components cannot be used to derive the stellar initial mass function. While current knowledge of the empirical mass-luminosity relation for masses more than $1.5 M_{\odot}$ is based exclusively on eclipsing binaries data, accurate observational data for a few hundred visual binaries of intermediate and high masses should be collected. The initial mass function for this mass range should then be revised.

\subsection{Satellite missions}

Söderhjelm continued studies on the double star observing capabilities of ESA's planned Gaia satellite. Although results may not be available until around 2020, one has to be aware of this coming (qualitative and quantitative) leap for double star astronomy. As summarized, e.g., in Söderhjelm (2005), Gaia will observe several hundred thousand eclipsing binaries, millions of astrometric pairs at 1-10 yr period, and tens of millions of longer-period binaries, thus sampling the general period-distribution over almost its full 
range. With accurate parallax distances for all the systems, we will get by then a much more complete picture of e.g. age- and place-variations of the distributions of orbit sizes and mass ratios, plus of course large numbers of accurate stellar masses.

\subsection{Theory of hierarchical star clusters}

Surdin (2004) has shown that hierarchical star clusters (H-clusters) containing tens of components may exist in the Galaxy. The number of stars $(N)$ in $\varepsilon$ Lyrae-type hierarchical systems is limited by the tidal action of regular gravitational field of the Galaxy and stochastic encounters with giant molecular clouds. In principle, the existence of $\mathrm{H}$-clusters with $N=256 \div 512$ is possible. But in fact, we can expect to find these systems with $N \leqslant 16$ in the recent astrometric surveys. Maximum fractal dimension of the simple stable H-cluster is $D=0.3 \div 0.4$, which is much less than the fractal dimension of molecular clouds $(D=2.3 \div 2.5)$. This may be the reason of rare formation of high populated H-systems.

\section{Working Group on Binary and Multiple System Nomenclature}

Following a Special Session held during the 2003 Sydney General Assembly, a Working Group (B. Mason, chair) was formed within Commission 26. Its purpose is to create the Washington Multiplicity Catalog, a comprehensive database of all known binaries. Data are being compiled from the USNO visual binary catalogs, as well as catalogs of spectroscopic, eclipsing, and interacting systems, plus extra-solar planets and other substellar companions. The goal is to create not only a comprehensive database but also a common nomenclature scheme to describe system hierarchy and to reduce confusion in the study of binaries using multiple techniques.

Statistics on multiplicity and apparent hierarchies of all known field binaries and multiples were recently generated from a draft version of the WMC; these results were presented at the Garching Multiple Stars Workshop in 2005 (Mason \& Hartkopf, submitted).

\section{Acknowledgements}

I should like to express my thanks to C. Allen, G. van Belle, T. ten Brummelaar, J. Davis, E. Horch, J. Kubat, P. Lampens, P. Lawson, O. Yu. Malkov, B. Mason, H. McAlister, E. Oblak, J.-L. Prieur, C. Scarfe, S. Söderhjelm, V. Tamazian, A. Tokovinin, N. Turner, and D. Wallace, all of whom kindly contributed to this report.

\section{References}

Allen, C., Poveda, A., \& Hernandez-Alcantara A. 2004, RevMexAA(SC), 21, 195

Boden. A. et al. 2005, BAAS, 206, 1419

Bouy, H. et al. 2004, A\&SA, 423, 341

Davis, J. et al. 2005, MNRAS, 356, 1362

Delfosse, X. et al. 2004, ASP Conf. Series, 318, 166

Duchene, G. et al. 2004, A\& $A, 427,651$

Frémat, Y., Lampens, P. \& Hensberge, H. 2005, MNRAS, 356, 545

Hartkopf, W.I. et al. 1999, AJ, 118, 509

Henry, T.J. 2004, ASP Conf. Series, 318, 159

Hensberge, H., Vaz, L.P.R. \& Torres, K.B. 2005, Proc. ESO Workshop "Multiple Stars Across the $H$ - $R$ Diagram", in preparation

Hill, G.M., Moffat, A.F.J., \& St.-Louis, N. 2002, MNRAS, 335, 1069

Hummel, C.A. 2004, ASP Conf. Series, 318, 13

Koubsky, P. et al. 2005, Proc. ESO Workshop "Multiple Stars Across the H-R Diagram", in preparation 
Kouwenhoven, M.B.N. et al. 2005, A\&A, 430, 137

Lampens, P. et al. 2005a, A\&BA, 438, 201

Lampens, P. et al. 2005b, Proc. ESO Workshop "Multiple Stars Across the H-R Diagram", in preparation

Malkov O. Yu. 2003, A\&A, 402, 1055

Mason, B.D. et al. 2004a, AJ, 127, 539

Mason, B.D. et al. 2004b, AJ, 128, 3012

Nelemans, G. et al. 2004, MNRAS, 349, 181

Oblak, E. et al. 2004, ASP Conf. Series, 318, 175

Patience, J. et al. 2002, ApJ, 581, 654

Patience, J., Akeson, R., \& Jensen, E. 2003, IAU Symp., 221, 83

Pourbaix, D., Jancart, S., \& Jorissen, A. 2004, ASP Conf. Series, 318, 144

Poveda, A. \& Allen, C. 2004, RevMexAA (SC), 21, 49-57

Richichi, A. 2004, ASP Conf. Series, 318, 148

Roberts, Jr., L.C. et al. 2005, AJ, in press

Scardia M. et al. 2005b, MNRAS, submitted

Scardia M. et al. 2005a, MNRAS, 357, 1255

Shatsky, N.I. \& Tokovinin, A.A. 2002, A\&A, 383, 92

Söderhjelm, S. 2005, Proc. "The three-dimensional universe with Gaia", ed. C. Turon, K.S. O'Flaherty, \& M.A.C. Perryman, ESA SP-576, 97

Surdin V.G. 2004, ASP Conf. Series, 316, 189

Tamazian, V. 2004, AJ, 127, 2378

Tamazian, V. et al. 2002, ApJ, 578, 925

Tokovinin, A.A. et al. 2003, A\&SA, 409, 245

Torres et al. 2002, AJ, 124, 1716

Turner, N.H., ten Brummelaar, T.A.,. \& Roberts,Jr., L.C. 2003, SPIE, 4839, 1103

Tuthill, P.G., Monnier, J.D., \& Danchi, W.C. 1999, Nature, 398, 487

Wallace, D.J. et al. 2005, AJ, 130, 126

Wallace, D.J. 2003, Ph.D. Dissertation, Georgia State University

Wallace, D.J. et al. 2001, in "The Influence of Binaries on Stellar Population Studies", AASL Ser. No. 264, ed. D. Vanbeveren (Dordrecht: Kluwer), 173

Williams, P.M., van der Hucht, K.A., \& The, P.S. 1987, A\&A A, 182, 91

W.I. Hartkopf

President of the Commission 